\title{
Processing Escapes: A New Perspective on Immune Escape Mechanisms
}

\author{
Michael Wessolly and Fabian Dominik Mairinger* \\ Institute of Pathology, University Hospital Essen, University of Duisburg-Essen, Essen, Germany
}

Submission: June 04, 2017; Published: June 08, 2017

"Correspondence Address: Fabian D Mairinger, AG "Translational Cancer Research", University Hospital Essen, Institute of Pathology, Hufelandstraße 55, D-45147 Essen, Tel: +49 (0) 177 / 843 1052; Cordless +49 (0) $201 / 723$ - 84828; Fax +49 (0) 201 / 723 - 3880 ;

Email: fabian.mairinger@uk-essen.de

\begin{abstract}
Novel approaches in cancer therapy have emerged in recent years. It is well-established that tumors tend to have a different antigenicity than normal cells, thereby triggering an immune response against the tumor, which include the development of tumor specific lymphocytes One distinct therapeutically approach involves the usage of monoclonal antibodies for immune checkpoint inhibition. CTLA-4 and PD-1/PDL1 have been investigated as useful targets in clinical trials.

In recent years, new immune escape mechanisms have been described. These mechanisms involve the complex intracellular machinery providing epitopes for CTLs. Important checkpoints of this process are the proteasomal degradation, the TAP- as well as the HLA-binding. It is hypothesized, proteasomal escape may play a role in developing resistance against this form of therapy. Deficient processing, either a defective or an extended epitope, can lead to non-recognition of those by CTLs. A weak binding of HLA: TCR would also prevent for example the PD-1/PDL-1 interaction. This would explain why some tumours are not susceptible to immunotherapy.
\end{abstract}

\section{Background - Cancer Therapy in the Course of Time}

With 14.1 million cases and 8.2 cancer related deaths worldwide, cancer is considered a global burden. From 2008 to 2012 the number of incidences and deaths has steadily raised. While the incidence in developed countries is about twice as high as in developing countries, the mortality rate does not differ that much $[1,2]$. It was estimated, that the incidence of cancer will rise from 12.7 to 22.2 million in 2030 [3]. Since it has been considered a global health problem, many kinds of new therapies have been developed over the last decades. Primary tumors alongside infested lymph nodes can be handled by surgical resection $[4,5]$. Although surgical resection has fallen behind other techniques like radio-or chemotherapy, it can be considered as the most effective and only real, possibly curative way to safely remove parts of the tumour. However, chemotherapeutic regimes as well as radiotherapy are also implicated complementary to surgical treatment. While operative techniques as well as the combined therapy have been improved, only $10-15 \%$ of metastatic cases can be approached surgically $[5,6]$.

$40 \%$ of Cancers are successfully therapied with ionizing radiation [5,7]. It can singly be applied or in combination with surgery and chemotherapy. Because of its good efficiency/cost ratio, it is already applied to $45 \%$ of new emerging cancers. Ionizing radiation uses electronically charged particles to damage DNA of tumour cells. While normal cells can also be harmed, they might repair themselves due to DNA damage repair, tumour cells lack this ability and are more likely forced into apoptosis [7,8]. Another frequently applied therapy is chemotherapy. Mechanisms of different chemotherapeutic agents vary greatly, ranging from induction of oxidative burst [9], DNA-damage $[10,11]$ or disruption of folic acid synthesis to inhibit cell division [4]. To improve on selectivity of radioand chemotherapy, the agents are conjugated with monoclonal antibodies [12].

\section{Immune Therapy}

Novel approaches in cancer therapy have emerged in recent years [13-15]. It is well-established that tumors tend to have a different antigenicity than normal cells, thereby triggering an immune response against the tumor, which include the development of tumor specific lymphocytes [13,16-18]. One subtype consists cytotoxic lymphocytes (CTLs). The interaction of these might result in the destruction of their target cells, ensured via release of perforin and granzymes as well as Fas-/FasL-mediated apoptosis [18,19]. Modern therapeutic 
approaches encompass vaccination, adaptive T-cell therapy as well as immune checkpoint inhibition via antibodies [13,20,21], (Figure 1A).

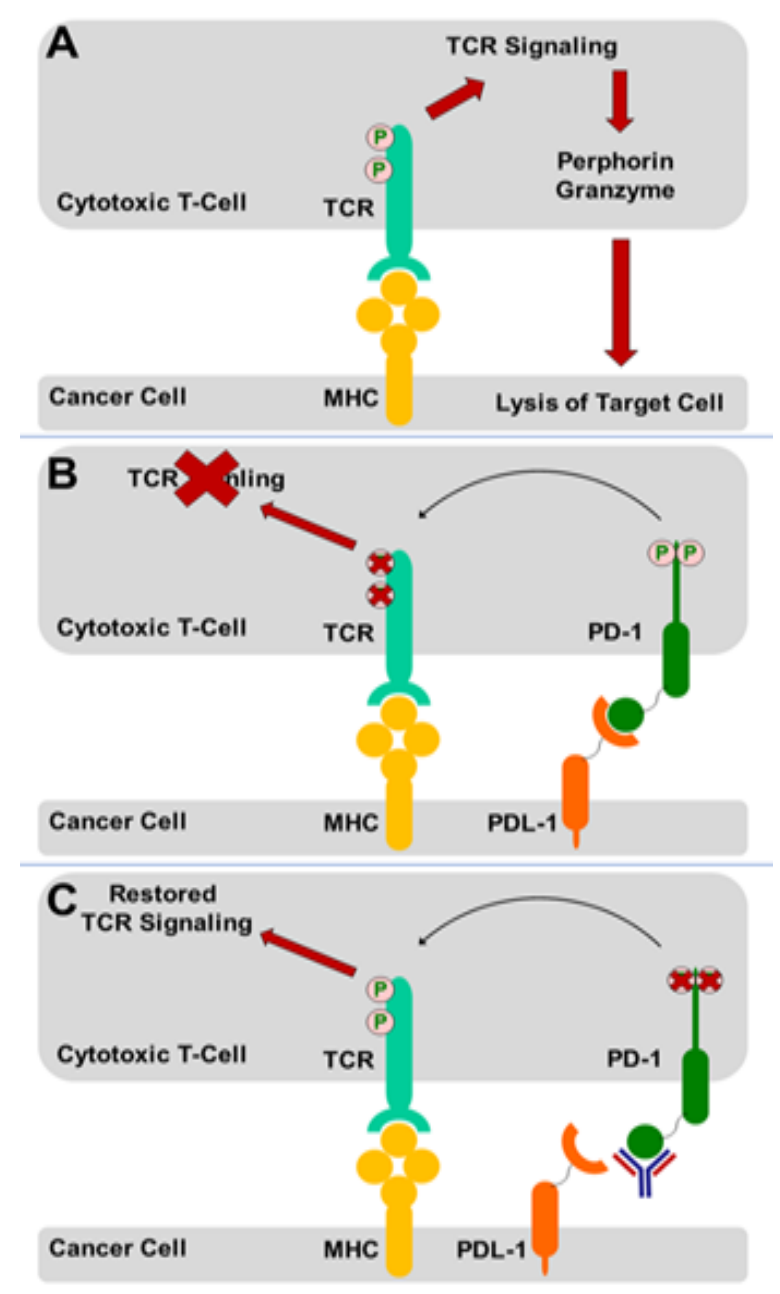

Figure 1: Antigen recognition through CTLs, TCR signalling and mechanism of action of immune checkpoint inhibitors.

A. CTLs bind the antigen-carrying MHC molecules on the target cells with their T-cell receptor (TCR). After recognition of a "foreign" antigen, the TCR get phosphorylated and the TCR signalling pathway gets initialized, resulting in secretion of perphorin and granzymes. Those lead to lysis of the target cell. B. Via overexpression of PDL-1 by cancer cells, the phosphorylation of PD-1 at the CTL and thereby lead to dephosphorylation of the TCR and result in a downregulation of T-cell response.

C. Through inhibition of PD-1/PDL-1 binding with anti-PD-1antibodies, TCR signalling gets restored and again leads to lysis of the target cell.

\section{Immune Checkpoint Inhibition}

One distinct therapeutically approach involves the usage of monoclonal antibodies for immune checkpoint inhibition. CTLA4 and PD-1/PDL-1 have been investigated in clinical trials [20,22]. CTLA-4 is known for counter regulation of co-stimulatory signals during T-cell activation [20]. $\alpha$ CTLA-4 antibodies are already applied for melanoma and mesothelioma therapy [23-25]. Two fully humanized monoclonal antibodies named Ipilimumab and Tremelimumab were developed and clinical tests started in 2000 [20]. Usage of Ipilimumab in melanoma therapy lead to a survival benefit of 3,5 months [25]. As result of this, it gained FDA approval in 2010, unlike Tremelimumab [20]. The latter one was also tested for mesothelioma therapy [23,24], but with minimal benefit [23]. As of January $24^{\text {th }}, 2017$ a double-blind study with Placebo und Tremelimumab usage is still ongoing (NCT01843374).

PD-1 is expressed on T-cells, while the ligand (PDL-1) is expressed on tumor cells. Their interaction is associated with downregulation of T-cell response [20], (Figure 1B). Nivolumab and Pembrolizumab are both monoclonal antibodies targeting PD-1 on CTLs (Figure 1C). Both have already been approved for usage in melanoma and squamous non-small cell lung carcinoma (NSCLC) [26]. In addition, Nivolumab was also applied in cases of platinum-resistant ovarian cancer and Hodgkin's lymphoma. Observed effects on progression-free survival were noticeable $[27,28]$. Application in renal cell carcinoma also improved overall survival [29]. Pembrolizumab has also been tested in various PDL-1 positive tumours like small cell lung carcinoma, mesothelioma, gastric cancer and oesophageal cancer [30-33]. All tests revealed a definite tumour response in a subgroup of patients.

Combination therapies with Ipilimumab ( $\alpha$ CTLA-4) and Nivolumab $(\alpha \mathrm{PD}-1)$ have also emerged. They are applied in metastatic melanoma with durable anti-tumour responses $[15,34,35]$. Several trails of Nivolumab, Ipilimumab and Pembrolizumab in combination with chemotherapeutic agents or combined antibodies are currently conducted in SCLC patients [36].

\section{Induction of T-cell response}

The activation of tumor specific T-cells occurs via presentation of small peptide fragments originating from tumor antigens $[37,38]$. A complex intracellular pathway is involved to process these antigenic peptides (Figure 2A). It starts with polyubiquitination of the protein, which is then degraded by the proteasome. The small fragments are further trimmed to an optimal length of 8-11 amino acids. Translocation of those into the ER is performed via the TAP-transporter, an ABC transporter (ATP-driven) composed of TAP1 and TAP2 (heterodimer) [39]. In the following, the peptide fragments bind the HLA class I molecule and the whole complex gets transported to the cell surface. Only peptide fragments bound by the specific HLA class I molecules are recognized [37,38]. Classically, three genes (HLA-A, HLA-B, HLA-C) with an ample number of alleles code for the HLA class I molecule, but also inferior genes are known [40]. Consequently, those have been reported to be one of the most polymorphic genes [40]. 


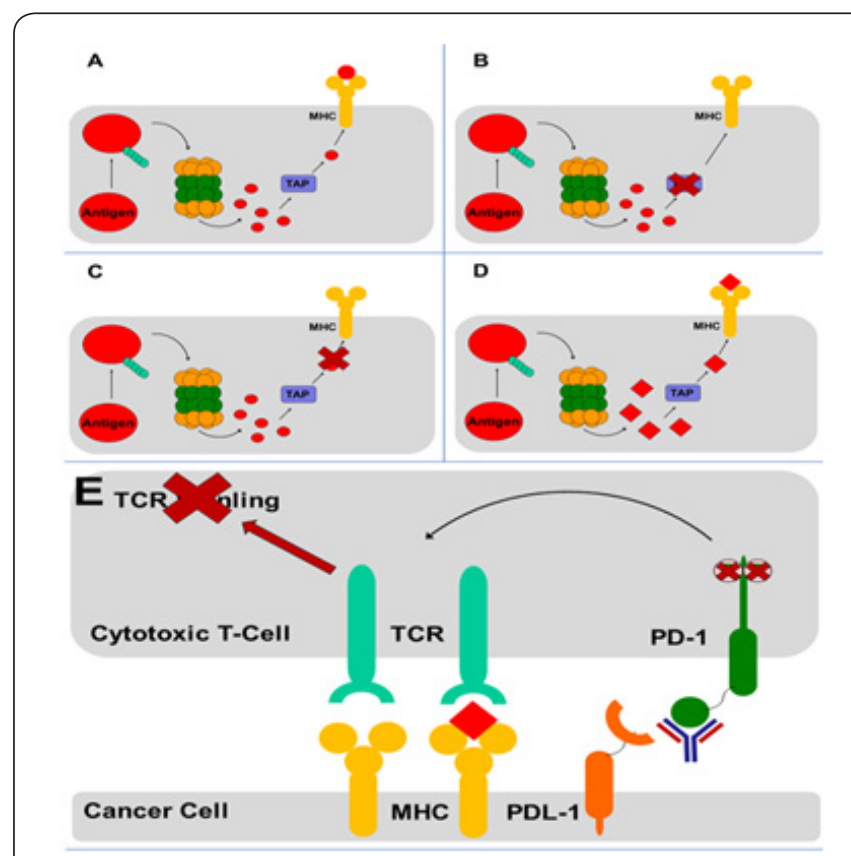

Figure 2: Overview of processing escape mechanisms.

A. A complex intracellular pathway is involved to process antigens, starting with polyubiquitination of the protein and subsequent degradation by the proteasome. Translocation of those fragments into the ER is performed via the TAPtransporter. In the following, the peptide fragments bind the HLA class I molecule and the whole complex gets transported to the cell surface. Processing escape mechanisms encompass.

B. Deficient binding to the TAP transporter,

C. Altered binding to $\mathrm{MHC}$ and subsequent transport to the cell surface as well as

D. Alternative proteasomal cleavage leading to differing peptides with varying lengths.

E. All mentioned mechanisms lead to either antigens not loaded to the MHC or antigens not recognized by the TCR, thereby leading to a resistance mechanism of a PD-1/PDL-1 blockade without restorage of TCR signalling pathways.

\section{Immune Response and Escape Mechanisms}

Considering darwinistic behaviour of pathogenic entities like viruses or tumours [41-46] it can be concluded, that the major part of the population has been wiped out during a selection event. However, some part of the population may have inherited favourable mutations helping to counteract selection pressure. Therefore, a new population is established, which might be even more aggressive towards the host [41-44,47]. Selection pressure includes antiviral agents, therapeutic agents against cancer as well as the immune response of the host [21,44-49].

\section{Immune Response at a Glance}

The immune response can be divided into two timely gated processes, the innate and the adaptive immune response. The innate response starts immediately after a pathogen is recognized as foreign [50]. During that time, pathogen fragments are loaded on specialized cell types called antigen presenting cells (APCs). These cells are the crucial link between both immune processes and are responsible for inducing the adaptive immune response
[50]. The process of adaptive response starts four days to one week after recognition of a pathogen [51]. The most well characterized cell types involved are T-and B-lymphocytes. B-lymphocytes are well known to produces antibodies, while T-lymphocytes can be divided into CD8 positive (CTLs) and CD4 positive T-lymphocytes, the latter having a supportive function for B-lymphocytes and CTLs (T-helper lymphocyte).

B-lymphocytes are mostly targeting important surface structures of pathogens (epitopes) [50-53]. Unfortunately, selection pressure favours mutation in these epitopes, leading to a reduced recognition of the target. The same goes for epitope recognition by CTLs.

\section{Processing Escapes}

In recent years, new immune escape mechanisms have been described. These mechanisms involve the complex intracellular machinery providing epitopes for CTLs. Important checkpoints of this process are the proteasomal degradation, the TAP- as well as the HLA-binding [21,39,48,54,55], (Figures 2B-2D). We decide to loosely term the escape mechanisms involved in the different checkpoints of epitope processing as processing escapes.

Johnsen et al. [56] observed the development of large and persistent tumours through TAP1-negative parental transformed murine fibroblast cell line. In case of tumour progression, TAP1negative cells have been reported to be selection-wise favoured over TAP1-positive cells [56]. In 1993 Restifo et al. [57] already suggested a possible tumour escape mechanism in relation to deficient antigen presentation and processing due to their finding of low mRNA levels for LMP-2 and LMP-7 (proteasome subunits) and TAP1 and TAP2 in small lung cell carcinomas (SCLC). In the review of Abele and Tampè (2011) [39], escape mechanisms involving TAP were described for both tumours and viruses, involving TAP-mutations and cofactors that interact with TAP.

Immune evasion from CTLs via deficiencies in the antigen presentation machinery is well described in different tumours $[55,58,59]$. These also include the deficiency of HLA/MHC class I molecules caused through point mutations or large deletions of HLA/MHC class I. Especially large deletions might result in loss of heterozygosity. However, subunits of HLA/MHC class I like Beta-2 micro globulin can also be affected by defective mutations [21,59]. In addition, tumours might also be capable of regulating HLA/MHC class I expression on an epigenetic level via DNA hypermethylation [60].

Immune escapes involving deficient proteasomal degradation is known in tumours [57,61], but especially in viruses like the hepatitis $C$ virus [48,62-64]. In this case not the epitope sequence itself, but especially the flanking region of epitopes is associated with the escape process. In 2016 Walker et al. [62] discovered a processing escape associated with the "highly conserved HLA-B*51 restricted epitope IPFYGKAI ${ }_{1373-1380}$ [62]. The investigated NS3-protein is multifunctional including a 
helicase as well as a serine protease activity. Because of its key role in RNA replication it is an important drug target, especially the protease domain [65]. Sequence analysis of multiple patient cohorts revealed a S1368P substitution five amino acids upstream of the epitopes $\mathrm{N}$-terminus.

Proteasomal processing of the mutated variant do not result in an impaired epitope. However, it is theorized, that the prolin substitution prevents N-terminal trimming $[66,67]$. Therefore, peptides of optimal size for epitope presentation are not generated, resulting in a less effective $\mathrm{T}$ cell response [62]. Another mechanism was described by Seifert et al. [63]. A different NS3-epitope (HLA-A*02-restricted) harboured a tyrosine/phenylalanine directly downstream of the C-terminal epitope position. Proteasomal processing result in a C-terminal extended epitope, which is also less effective in triggering an immune response [63]. It seems that mutations affecting processing at the $\mathrm{C}$-terminal side more likely result in an impairment of antigen processing. While $\mathrm{N}$-terminal elongated peptides might be further trimmed, the epitope defining cut is made at its C-terminus $[38,68,69]$. A somewhat similar mechanism was also found in HIV [64]. Three distinct mutations, both in the $\mathrm{N}$-and $\mathrm{C}$-terminal flanking region, were found in the HLA B*35-restricted Nef-epitope VLPRPMTY. If all three mutations were present it results in an alternative cleavage within the epitope. Thereby, a partially destroyed epitope is generated. While the impaired epitope was presented and successfully recognized, infected cells presenting it induce a less effect CTL response with low amounts of Interferon-gamma and less induced apoptosis. The same mechanism of differing epitope lengths may be present in neoplastic malignancies.

\section{Resistance to Immune Checkpoint Inhibitors via Processing Escapes}

While checkpoint inhibitors ( $\alpha \mathrm{PD} 1 / \alpha \mathrm{PDL} 1 / \alpha \mathrm{CTLA}-4)$ for immune responses have proven useful in about 15 different cancer types, $60 \%$ of patients are affected by inefficient response rates. In addition, some patients seem to develop some form of acquired resistance. Initial response showed some success, which was later undone due to acquired resistance [70].

It is hypothesized; proteasomal escape may play a role in developing resistance against this form of therapy. Deficient processing, either a defective or an extended epitope similar to viruses, can lead to non-recognition of those by CTLs (Figure 2E). A weak binding of HLA: TCR would also prevent for example the PD-1/PDL-1 interaction. This would explain why some tumours are not susceptible to immunotherapy. Even if antibodies would bind to their respective target it would have no effect, rendering the immune therapy useless. With this mechanism proposed, it is hoped to suggest an alternative immune escape, which might be worth investigating in the future. In addition, it remains to be investigated if processing escapes of some kind have a major impact on developing therapy resistance.

\section{Conflicts of Interest}

All authors state that they have no conflicts of interest to declare.

\section{References}

1. Jemal A, Bray F, Center MM, Ferlay J, Ward E, et al. (2011) Global cancer statistics. CA Cancer J Clin 61(2): 69-90.

2. Torre LA, Bray F, Siegel RL, Ferlay J, Lortet-Tieulent J, et al. (2012) Global cancer statistics. CA Cancer J Clin 65(2): 87-108.

3. Bray F, Ahmedin Jemal, Nathan Grey, Jacques Ferlay, David Forman (2012) Global cancer transitions according to the Human Development Index (2008-2030): a population-based study. Lancet Oncol 13(8): 790-801.

4. Gustavsson B, Carlsson G, Machover D, Petrelli N, Roth A, et al. (2015) A review of the evolution of systemic chemotherapy in the management of colorectal cancer. Clin Colorectal Cancer 14(1): 1-10.

5. Urruticoechea A, Alemany R, Balart J, Villanueva A, Viñals F, et al. (2010) Recent advances in cancer therapy: an overview. Current pharmaceutical design. 16(1): 3-10.

6. Ohlsson B, U Stenram, KG Tranberg (1998) Resection of colorectal liver metastases: 25-year experience. World journal of surgery 22(3): 268277.

7. Delaney G, Jacob S, Featherstone C, Barton M (2005) The role of radiotherapy in cancer treatment. Cancer 104(6): 1129-1137.

8. Baskar R, Lee KA, Yeo R, Yeoh KW (2012) Cancer and radiation therapy: current advances and future directions. Int J Med Sci 9(3): 193-199.

9. Fuchs-Tarlovsky V (2013) Role of antioxidants in cancer therapy. Nutrition 29(1): 15-21.

10. Dasari S, PB Tchounwou (2014) Cisplatin in cancer therapy: molecular mechanisms of action. Eur J pharmacol 740: 364-378.

11. Matsuoka A, Y Ando (2017) Nephropathy in Patients Undergoing Cancer Drug Therapy-Platinum Derivatives (Cisplatin and Carboplatin). Gan to kagaku ryoho 44(3): 200-203.

12. Padma VV (2015) An overview of targeted cancer therapy. Biomedicine 5(4): 19.

13. Brahmer JR, DM Pardoll (2013) Immune checkpoint inhibitors: making immunotherapy a reality for the treatment of lung cancer. Cancer immunology research 1(2): 85-91.

14. Topalian SL, Hodi FS, Brahmer JR, Gettinger SN, Smith DC, et al. (2012) Safety, activity, and immune correlates of anti-PD-1 antibody in cancer. N Engl J Med 366(26): 2443-2454.

15. Wolchok JD, Harriet Kluger, Margaret K Callahan, Michael A Postow, Naiyer A, et al. (2013) Nivolumab plus ipilimumab in advanced melanoma. New England Journal of Medicine 369(2): 122-133.

16. Rizvi NA, Hellmann, Snyder A, Kvistborg P, Makarov V, et al. (2015) Mutational landscape determines sensitivity to PD-1 blockade in nonsmall cell lung cancer. Science 348(6230): 124-128.

17. Adam JK, B Odhav, KD Bhoola (2003) Immune responses in cancer. Pharmacol Ther 99(1): 113-132.

18. Pandolfi F, R Cianci, D Pagliari, F Casciano, C Bagalà, et al. (2011) The immune response to tumors as a tool toward immunotherapy. Clinical and Developmental Immunology 2011: 12.

19. Paganelli R, Giovannetti A, Pierdominici M, Di Iorio A, Cianci R, et al. (2008) Apoptosis in the homeostasis of the immune system and in human immune mediated diseases. Curr pharm Des 14(3): 253-268. 
20. Pardoll DM (2012) The blockade of immune checkpoints in cancer immunotherapy. Nat Rev Cancer 12(4): 252-264.

21. Topfer K, Stefanie Kempe, Nadja Muller, Marc Schmitz, et al. (2011) Tumor evasion from $\mathrm{T}$ cell surveillance. BioMed Research International 2011: 19.

22. Zou W, L Chen (2008) Inhibitory B7-family molecules in the tumour microenvironment. Nature reviews. Immunology 8(6): 467-477.

23. Calabrò L, Aldo Morra, Ester Fonsatti, Ornella Cutaia, et al. (2013) Tremelimumab for patients with chemotherapy-resistant advanced malignant mesothelioma: an open-label, single-arm, phase 2 trial. The Lancet Oncology 14(11): 1104-1111.

24. Calabrò L, Morra A, Fonsatti E, Cutaia O, Fazio C, et al. (2015) Efficacy and safety of an intensified schedule of tremelimumab for chemotherapyresistant malignant mesothelioma: an open-label, single-arm, phase 2 study. Lancet Respir Med 3(4): 301-309.

25. Hodi FS, O Day SJ, McDermott DF, Weber RW, Sosman JA, et al. (2010) Improved survival with ipilimumab in patients with metastatic melanoma. N Engl J Med 363(8): 711-723.

26. Festino L, Botti G, Lorigan P, Masucci GV, Hipp JD, et al. (2016) Cancer treatment with anti-PD-1/PD-L1 agents: is PD-L1 expression a biomarker for patient selection? Drugs 76(9): 925-945.

27. Hamanishi J, Masaki Mandai, Takafumi Ikeda, Manabu Minami, Atsushi Kawaguchi, et al. (2015) Durable tumor remission in patients with platinum-resistant ovarian cancer receiving nivolumab. American Society of Clinical Oncology 33(15).

28. Ansell SM, Alexander M Lesokhin, Ivan Borrello, Ahmad Halwani, et al. (2015) PD-1 blockade with nivolumab in relapsed or refractory Hodgkin's lymphoma. New England Journal of Medicine 372(4): 311 319

29. Motzer RJ, Bernard Escudier, David F McDermott, Saby George, Hans J Hammers, et al. (2015) Nivolumab versus everolimus in advanced renal-cell carcinoma. New England Journal of Medicine 373(19): 18031813.

30. Toshihiko Doi, Sarina Anne Piha-Paul, Shadia Ibrahim Jalal, Hieu Mai-Dang, Sammy Yuan, et al. (2015) Pembrolizumab (MK-3475) in patients (pts) with extensive-stage small cell lung cancer (SCLC) Preliminary safety and efficacy results from KEYNOTE-028. American Society of Clinical Oncology 33(15).

31. Alley EW, Rhoda Molife, Armando Santaro, Kim Beckey, Sammy Yuan, et al. (2015) Abstract CT103: Clinical safety and efficacy of pembrolizumab (MK-3475) in patients with malignant pleura mesothelioma: Preliminary results from KEYNOTE-028. AACR 75(15).

32. Toshihiko Doi, Sarina Anne Piha-Paul, Shadia Ibrahim Jalal, Hieu Mai-Dang, Sammy Yuan, et al. (2015) Pembrolizumab (MK-3475) for patients (pts) with advanced esophageal carcinoma: Preliminary results from KEYNOTE-028. 2015, American Society of Clinical Oncology 33(15).

33. Muro K, Yung J Bang, Veena Shankaram, Hyun Cheol Chung, Ravit Geva, et al. (2015) Relationship between PD-L1 expression and clinical outcomes in patients (Pts) with advanced gastric cancer treated with the anti-PD-1 monoclonal antibody pembrolizumab (Pembro; MK3475) in KEYNOTE-012. 2015, American Society of Clinical Oncology 2015: 33.

34. Larkin J, Hodi FS, Wolchok JD (2015) Combined nivolumab and ipilimumab or monotherapy in untreated melanoma. N Engl J Med 373: 23-34.

35. Antonia SJ, Scott N Gettinger, Laura Quan Man Chow, Rosalyn A Juergens, Yun Shen, et al. (2014) Nivolumab (anti-PD-1; BMS-936558, ONO-4538) and ipilimumab in first-line NSCLC: Interim phase I results. American Society of Clinical Oncology 32(15).
36. Paglialunga L, Zena Salih, Biago Ricciuti, Raffaele Califano (2016) Immune checkpoint blockade in small cell lung cancer: is there a light at the end of the tunnel? ESMO open 1(4): e000022.

37. Blum JS, PA Wearsch PA, P Cresswell (2013) Pathways of antigen processing. Annual review of immunology 31: 443-473.

38. Kloetzel PM (2004) The proteasome and MHC class I antigen processing. Biochimica et Biophysica Acta 1695(1-3): 225-233.

39. Abele R, R Tampé (2011) The TAP translocation machinery in adaptive immunity and viral escape mechanisms. Essays in biochemistry 50(1): 249-264.

40. Choo SY (2007) The HLA system: genetics, immunology, clinical testing, and clinical implications. Yonsei medical journal 48(1): 11-23.

41. Pepper JW, C Scott Findlay, Rees Kassen, SL Spencer, CC Maley (2009) Cancer Research Meets Evolutionary Biology. Evolutionary Applications 2(1): 62-70.

42. Cairns J (1975) Mutation selection and the natural history of cancer. Nature 255(5505): 197-200.

43. Nowell PC (1976) The clonal evolution of tumor cell populations. Science 194(4260): 23-28.

44. Michor F, MA Nowak, Y Iwasa (2006) Evolution of resistance to cancer therapy. Current pharmaceutical design 12(3): 261-271.

45. Strasfeld L, S Chou (2010) Antiviral drug resistance: mechanisms and clinical implications. Infectious disease clinics of North America 24(2): 413-437.

46. Hayden FG (2006) Antiviral resistance in influenza virusesimplications for management and pandemic response. New England Journal of Medicine 354(8): 785-788.

47. Landau DA, Carter SL, Stojanov P, Mc Kenna A, Wanq L, et al. (2013) Evolution and impact of subclonal mutations in chronic lymphocytic leukemia. Cell 152(4): 714-726.

48. Rosenberg W (1999) Mechanisms of immune escape in viral hepatitis. Gut 44(5): 759-764.

49. Wölfl M (2008) Hepatitis C virus immune escape via exploitation of a hole in the T cell repertoire. The Journal of Immunology 181(9): 64356446.

50. Goldman L, AI Schafer (2011) Goldman's Cecil medicine. Elsevier Health Sciences.

51. Janeway CA (1997) Immunobiology: the immune system in health and disease Vol. 1 Current Biology. Singapore.

52. Rhorer J, Ambrose CS, Dickinson S, Hamilton H, Oleka NA, et al. (2009) Efficacy of live attenuated influenza vaccine in children: a metaanalysis of nine randomized clinical trials. Vaccine 27(7): 1101-1110.

53. Klasse PJ (2014) Neutralization of virus infectivity by antibodies: old problems in new perspectives. Advances in biology 2014(2014): 24

54. Sanda MG (1995) Molecular characterization of defective antigen processing in human prostate cancer. Journal of the National Cancer Institute 87(4): 280-285.

55. Maleno I, cabrera CM, Cabrera T, Paco L, Garrido F, et al. (2004) Distribution of HLA class I altered phenotypes in colorectal carcinomas: high frequency of HLA haplotype loss associated with loss of heterozygosity in chromosome region $6 \mathrm{p} 21$. Immunogenetics 56(4): 244-253.

56. Johnsen A (1999) Deficiency of transporter for antigen presentation (TAP) in tumor cells allows evasion of immune surveillance and increases tumorigenesis. The Journal of Immunology 163(8): 42244231. 
57. Restifo NP, esquivel F, Kawakami Y, Yewdwll JW, Mule JJ, et al. (1993) Identification of human cancers deficient in antigen processing Journal of Experimental Medicine 177(2): 265-272.

58. Maleno I, Cabrera T, Paco L, Tallada M, Cozar JM, et al. (2006) LOH at $6 \mathrm{p} 21.3$ region and HLA class altered phenotypes in bladder carcinomas. Immunogenetics 58(7): 503-510.

59. Bicknell DA, Rowan W, Bodmer (1994) Beta 2-microglobulin gene mutations: a study of established colorectal cell lines and fresh tumors. Proceedings of the National Academy of Sciences 91(11): 4751-4755.

60. Serrano AI, Castro-Vega I, M Redondo (2011) Role of gene methylation in antitumor immune response: implication for tumor progression. Cancers 3(2): 1672-1690.

61. Garcia-Lora, AI Algarra, F Garrido (2003) MHC class I antigens, immune surveillance, and tumor immune escape. Journal of cellular physiology 195(3): 346-355

62. Walker A, Skibbe K, Allen TM, Timm J, Lauer GM, et al. (2016) Distinct escape pathway by hepatitis $C$ virus genotype 1 a from a dominant CD8+ $\mathrm{T}$ cell response by selection of altered epitope processing Journal of virology $90(1): 33-42$

63. Seifert U, Liermann H, Racanelli V, Wiese M, Hengal H, et al. (2004) Hepatitis $\mathrm{C}$ virus mutation affects proteasomal epitope processing. The Journal of clinical investigation 114(2): 250-259.
64. Milicic A, Price DA, Zibwa P, Booth BL, Olsen K, et al. (2005) CD8+ T cell epitope-flanking mutations disrupt proteasomal processing of $\mathrm{HIV}-1$ Nef. The Journal of Immunology 175(7): 4618-4626.

65. Pang PS, Jankowsky E, Planet PJ, Pylee AM (2002) The hepatitis C viral NS3 protein is a processive DNA helicase with cofactor enhanced RNA unwinding. The EMBO Journal 21(5): 1168-1176.

66. Hammer GE, T Kanaseki, N Shastri (2007) The final touches make perfect the peptide-MHC class I repertoire. Immunity 26(4): 397-406.

67. Serwold T (2002) ERAAP customizes peptides for MHC class I molecules in the endoplasmic reticulum. Nature 419(6906): 480-483

68. Niedermann G, Butz S, Grimm R, Jung G, Maier B, et al. (1995) Contribution of proteasome-mediated proteolysis to the hierarchy of epitopes presented by major histocompatibility complex class I molecules. Immunity 2(3): 289-299.

69. Craiu A (1997) Two distinct proteolytic processes in the generation of a major histocompatibility complex class I-presented peptide. Proceedings of the National Academy of Sciences 94(20): 1085010855.

70. O'Donnell JS (2017) Resistance to PD1/PDL1 checkpoint inhibition. Cancer Treatment Reviews 52: 71-81.

\section{Your next submission with Juniper Publishers will reach you the below assets}

- Quality Editorial service

- Swift Peer Review

- Reprints availability

- E-prints Service

- Manuscript Podcast for convenient understanding

- Global attainment for your research

- Manuscript accessibility in different formats

( Pdf, E-pub, Full Text, Audio)

- Unceasing customer service

Track the below URL for one-step submission https://juniperpublishers.com/online-submission.php 